\title{
Editorial
}

\section{Dear Authors and Readers,}

With the present third issue (3-2014) of our e-Journal „International Dialogues on Education: Past and Present" we close the year 2014 and are getting ready for editing articles for the next issue (12015), which will be published in March 2015.

In view of the existing interests, for the next issue (1-2015) we will keep with the wide range of thematic orientation towards educational developments from historical, international-comparative and intercultural perspectives. However, a thematic special issue will also be forthcoming in August 2015 in cooperation with Seattle Pacific University (Seattle, USA), focused on historical and contemporary innovations in the education field (see also: http://davidwicks.org/iste-5-engage-inprofessional-growth-and-leadership/symposium-educational-innovations-in-countries-aroundthe-world/)

Another new addition is that we are now featuring academic essays (IDE Academic Essays) and are accepting submissions in this category. With this, we would like to encourage scholars to submit manuscripts that are not primarily based on empirical or theoretical studies but rather are centered on reflective or auto/biographical experiences related to research practices.

Dear authors and readers, we continue to strive to take a stand and act against the growing commercialization of access to scientific publications. However, in light of the increasing editorial, organizational, and technical workload associated with an international, intercultural and multilingual journal such as ours, it is necessary for us to charge a processing fee of 30 Euros per article, beginning with the 1-2015 issue. The submission of manuscripts to be considered for peer review as well as reviews will continue to be cost-free. Suitable articles will be published after receipt of the processing fee.

We look forward to further high-quality manuscripts concerning educational research and praxis in all these contexts. We especially ask all authors of articles to adhere to our formal guidelines, which can be accessed at http://www.ide-journal.org/instructions-to-contributors/.

Best wishes for all of your continued success.

The Editors

http://www.ide-journal.org/editorial-board/

http://www.ide-journal.org/contacts/

\section{Дорогие авторы, дорогие читатели,}

настоящим третьим номером (3-2014) нашего электронного журнала «Интернациональные диалоги в образовании: прошлое и настоящее» мы завершаем 2014 год и готовимся к редакционной работе со статьями для следующего номера (1-2015), который выйдет в свет в марте. 
Ввиду актуального интереса мы сохраняем для следующего номера (1-2015) широкую тематическую ориентацию на развитие образования в историческом, международносравнительном и межкультурном направлении. Однако в сотрудничестве с Тихоокеанским университетом г. Сиэтл (Сиэтл, США) в августе 2015 года выйдет специальный тематический номер, и будет концентрироваться на исторических и актуальных инновациях в области образования. (см. также: http://davidwicks.org/iste-5-engage-inprofessional-growth-and-leadership/symposium-educational-innovations-in-countries-aroundthe-world/)

Другое новшество состоит в том, что мы начинаем принимать категорию манускриптов в виде научных эссе (IDE Academic Essay). Этим мы хотим пробудить в ученых интерес к предложению манускриптов, которые, в первую очередь, не на эмпирических или теоретических исследованиях, а выдвигают в центр внимания опыт осмысления и биографический опыт, вытекающий из практики исследования.

Дорогие авторы, дорогие читатели, мы, как и прежде, прилагаем все усилия к тому, чтобы бороться против коммерциализации научных публикаций. Но ввиду возросших редакционных, организационных и технических затрат в таком международном, межкультурном и многоязыковом журнале как наш, мы не избежим того, чтобы, начиная с номера 1-2015 мы предусматриваем оплату за редакционную обработку в размере 30 евро за статью. Предъявление манускриптов для коллегиальной проверки, а также рецензии остаются, как и прежде, бесплатными. Статьи, принятые к публикации, будут опубликованы после поступления оплаты.

Мы всегда рады качественно убедительным манускриптам, которые волнуют науку и практику образования. Мы убедительно просим авторов статей следовать формальным рекомендациям, которые находятся по адресу: http://www.ide-journal.org/instructions-tocontributors/.

\section{Редакция желает Вам больших успехов!}

http://www.ide-journal.org/editorial-board/

http://www.ide-journal.org/contacts/

\section{Liebe Autorinnen und Autoren, liebe Leserinnen und Leser,}

mit der nun vorliegenden dritten Ausgabe (3-2014) unseres e-Journals „International Dialogues on Education: Past and Present" schließen wir das Jahr 2014 ab und bereiten uns auf die redaktionelle Bearbeitung von Artikeln für die nächste Ausgabe (1-2015) vor, die im März erscheinen wird.

Angesichts der aktuellen Interessenlage behalten wir auch für die nächste Ausgabe (1-2015) die weite thematische Orientierung auf Bildungsentwicklungen in historischer, internationalvergleichender und interkultureller Perspektive bei. Es wird aber auch eine thematisch fokussierte Extra-Ausgabe in Kooperation mit der Seattle Pacific University (Seattle, USA) geben, die im August 2015 erscheinen wird, und die sich auf historische und aktuelle Innovationen im Bildungsbereich konzentriert (siehe auch: http://davidwicks.org/iste-5-engage-in-professional-growth-andleadership/symposium-educational-innovations-in-countries-around-the-world/).

Ein weiteres Novum besteht darin, dass wir ab sofort auch die Manuskriptkategorie des wissenschaftlichen Essays (IDE Academic Essay) aufnehmen. Damit wollen wir Wissenschaftler 
anregen, auch Manuskripte einzureichen, die nicht in erster Linie auf empirischen und theoretischen Studien basieren, sondern reflektierte und auto/biographische Erfahrungen aus der Forschungspraxis in den Mittelpunkt stellen.

Liebe Autorinnen und Auroren, liebe Leserinnen und Leser, wir bemühen uns nach wie vor, einer umsich greifenden Kommerzialisierung des Zugangs zu wissenschaftlichen Publikationen entgegenzuwirken. Angesichts des gewachsenen editorischen, organisatorischen und technischen Bearbeitungsaufwandes bei einem derart internationalen, interkulturellen und mehrsprachigen Journal wie dem unseren, kommen aber auch wir nicht umhin, beginnend mit der Ausgabe 1-2015 eine Bearbeitungsgebühr in Höhe von 30 Euro pro Artikel zu berücksichtigen. Das Einreichen von Manuskripten zur Prüfung im Peer Review-Verfahren sowie Rezensionen bleiben auch weiterhin gebührenfrei. Publikationsfähige Artikel werden veröffentlicht, wenn die Bearbeitungsgebühr eingegangen ist.

Wir freuen uns auf weitere, qualitativ überzeugende Manuskripte, die die Bildungsforschung und Bildungspraxis bewegen. Wir bitten die Autoren von Artikeln ausdrücklich um Beachtung der formalen Empfehlungen unter http://www.ide-journal.org/instructions-to-contributors/.

\section{Die Redaktion wünscht Ihnen viel Erfolg!}

http://www.ide-journal.org/editorial-board/ http://www.ide-journal.org/contacts/ 